\title{
Concurrent performance of motor tasks and processing capacity in patients with schizophrenia
}

\author{
Rebecca Fuller, Marjan Jahanshahi
}

\begin{abstract}
Any task is carried out more successfully if we allocate undivided attention to it, but as demands on attentional capacity increase-for example, in concurrent or dual task conditions-performance on attended tasks becomes more impaired. Patients with schizophrenia show impaired performance on tasks requiring high levels of attentional capacity. This study examines performance of 11 patients with schizophrenia and 13 normal controls on two motor tasks (placing pegs in a pegboard and repetitive index finger tapping) under unimanual, bimanual, and dual task conditions. The patients with schizophrenia placed fewer pegs and had reduced tapping speed in unimanual and bimanual conditions. However, the decrement in bimanual performance as a percentage of unimanual performance was not significantly different for the patients and controls on either the pegboard or tapping tasks. By contrast, under dual task conditions, the performance of the patients with schizophrenia in peg placement actually improved relative to the unimanual pegboard task, whereas tapping performance deteriorated compared with the unimanual tapping, a decrement that was significantly greater for the patients. Thus the improvement in the visually guided pegboard task was at the expense of the repetitive tapping task. These results are discussed in terms of an impairment of self initiated movement with general sparing of externally triggered movements in schizophrenia and the role of frontostriatal loops in this process.
\end{abstract}

(F Neurol Neurosurg Psychiatry 1999;66:668-671)

Keywords: schizophrenia; dual task; processing resources; finger tapping; Purdue pegboard

Any task is carried out more successfully if we allocate our undivided attention to it. As demands on attentional capacity increase-for example, in concurrent or dual task conditions-performance on attended tasks becomes more impaired. ${ }^{1}$ The degree to which a task is impaired, however, depends on the amount of attentional capacity it demands. An automatic task would not interfere with a more demanding task but there would be interference of one very demanding task on another. ${ }^{2}$

Schizophrenia is a psychiatric disorder characterised by positive symptoms (for example, hallucinations and delusions) and negative signs (for example, flattening of affect, poverty of speech, and social withdrawal). ${ }^{3}$ It has been suggested that as cognitive load increases, patients with schizophrenia show greater task impairment than normal subjects, signifying resource limitations in this disorder. ${ }^{4}$ One way to test the effects of increasing cognitive load is to compare performance on dual task with single task conditions. ${ }^{5}$ It would be expected that the degree of performance decrement in a dual task compared with a single task condition would be greater in patients with schizophrenia than in controls, due to the patients' limited resources and hence inability to adequately handle the increased cognitive load. There is evidence that under dual task conditions patients with schizophrenia are differentially more impaired than normal subjects $^{6}$ and other psychiatric patients. ${ }^{78}$ These studies ${ }^{6-8}$ used a simple reaction time task and tests of cognitive skill, such as a visual search task or counting.

Another way to investigate attentional processing capacity in schizophrenia is to assess concurrent performance of motor tasks such as finger tapping and peg placement, which differ in terms of their attentional demands. Peg placement as in the Purdue pegboard task involves sequential movements such as grasping and picking a peg up, followed by its transport and insertion into the holes on the pegboard. Because of its visually driven nature, performance on the pegboard seems to gain prominence over repetitive index finger tapping, which can be performed in a more automatic fashion. As a result, in normal subjects, when performed concurrently, finger tapping interferes relatively less with peg placement, whereas concurrent peg placement affects finger tapping more. ${ }^{9}$

The aim of this study was to examine processing resources in schizophrenia by comparing the performance of two motor tasks, tapping and pegboard placement, under unimanual, bimanual, and dual task conditions in patients with schizophrenia and normal controls. 
Performance of the two groups on the tapping and Purdue pegboard tasks (mean (SD)) under unimanual, bimanual, and dual task conditions

\begin{tabular}{|c|c|c|c|c|}
\hline & Schizophrenic patients & Normal controls & p Value & ${ }^{*} p$ Value \\
\hline Pegboard unimanual & $13.1(2.3)$ & $16.3(1.8)$ & $<0.01$ & $<0.05$ \\
\hline Pegboard bimanual & $11.3(2.8)$ & $14.1(1.7)$ & $<0.01$ & 0.08 \\
\hline$\%$ Of unimanual pegboard & $85.4(10.1)$ & $87.2(7.6)$ & 0.53 & 0.74 \\
\hline Tapping unimanual & $145.5(40.0)$ & $172.6(19.1)$ & 0.06 & 0.19 \\
\hline Tapping bimanual & $132.8(38.7)$ & $165.2(26.4)$ & $<0.03$ & 0.26 \\
\hline$\%$ Of unimanual tapping & $92.7(15.0)$ & $94.2(9.8)$ & 0.77 & 0.55 \\
\hline Pegboard with tapping & $13.3(2.5)$ & $15.0(2.1)$ & $<0.04$ & 0.30 \\
\hline$\%$ Of unimanual pegboard & $101.3(5.2)$ & $92.5(8.7)$ & $<0.02$ & 0.10 \\
\hline Tapping with pegboard & $100.8(38.3)$ & $149.6(22.3)$ & $<0.01$ & 0.07 \\
\hline$\%$ Of unimanual tapping & $71.2(22.9)$ & $86.8(9.8)$ & 0.05 & 0.26 \\
\hline PEGu-PEGc & & & & \\
\hline$\%$ Subjects showing improvement & 45.5 & 15.4 & \multirow{3}{*}{\multicolumn{2}{|c|}{$\mathrm{X}^{2}(2)=5.92 \mathrm{p}=0.05$}} \\
\hline$\%$ Subjects showing no change & 27.3 & 7.7 & & \\
\hline$\%$ Subjects showing deterioration & 27.3 & 76.9 & & \\
\hline
\end{tabular}

^Results of one way ANCOVA with MMS and BDI score as covariates.

\section{Method}

SUBJECTS

Eleven (two female, nine male) subjects with a clinical diagnosis of schizophrenia according to the DSM IIIR criteria were tested. Each was seen as an outpatient at the National Hospital for Neurology and Neurosurgery. Each patient was rated on the Krawiecka Manchester scale, ${ }^{10}$ a four point standardised psychiatric assessment scale for current positive and negative symptoms. The mean score for positive symptoms (incoherence, delusions, and hallucinations) was 2.0 and the mean score for negative signs (poverty of speech, flattened affect, and psychomotor retardation) was 2.2. The mean age for the patients was 38.5 (range 27-55). As a group, the patients with schizophrenia were chronically ill, with a mean duration of illness of 14.2 (SD 7.4) years. All patients except two were on medication with nine on neuroleptic drugs (mean dose 375.6 (SD 142.3) mg. Thirteen (five female, eight male) healthy normal subjects with no history of psychiatric or neurological illness, head injury, or drug misuse were tested. Their mean age was 38.15 (range 21-62). All subjects were right handed. To screen for cognitive deficits, the mini mental state examination ${ }^{11}$ was administered to all subjects. None of the subjects scored below the cut off point indicative of cognitive deficit.

PROCEDURE

Detailed descriptions of procedures are given by Brown et al. ${ }^{12}$ All subjects performed finger tapping and the Purdue pegboard task under unimanual, bimanual, and dual task conditions. In this task, subjects are required to place metal pegs $(3 \mathrm{~mm} \times 25 \mathrm{~mm})$ in a vertical row of holes, as quickly as possible for a 30 second period. Subjects performed the task with each hand separately, and then bimanually. The measures of unimanual and bimanual performance were the mean number of pegs placed with the left and right hands under each condition.

The second task was repetitive index finger tapping. Subjects were required to continuously tap a $25 \mathrm{~mm}$ button as quickly as possible for a 30 second period. The button activated a $150 \mathrm{~g}$ standard microswitch. The task was performed with each hand separately and bimanually. As with the pegboard, the measures of unimanual and bimanual performance were the mean number of taps with the left and right hands under each condition.

Subjects also performed a combined bimanual task. This involved tapping with one hand and placing pegs with the other. The task was performed twice, once with each hand-task combination each time for 30 seconds. The average of the two tests was calculated for each task. For all conditions the measures of bimanual performance were expressed as percentages of unimanual performance (for example, $(\mathrm{PEGb} / \mathrm{PEGu}) \times 100)$. In the combined task condition, subjects were instructed to perform both tasks at the same time as fast as they could and not to concentrate on one to the exclusion of the other.

The order of testing was randomised across subjects. All subjects were assessed on the Beck depression inventory (BDI). ${ }^{13}$

\section{Results}

The mean ages of the two groups did not differ significantly $(F(1,23)=0.032, \mathrm{p}=0.87)$. The mean of the MMS scores for the patients was 28.2 (SD 2.5) and for the controls 29.9 (SD $0.3)$. The difference between the two groups was significant $(F(1,23)=6.35, \mathrm{p}=0.02)$. The mean score on the BDI for the patients was $14.4(\mathrm{SD}=10.3)$ and for the controls it was 4.5 (SD 5.1), a difference which was significant $(F(1,22)=9.20, \mathrm{p}<0.01)$. The group differences in task performance were analysed both with and without MMS and BDI scores as covariates.

The table shows the mean performance of the two groups for each of the test conditions, as well as the performance of the bimanual conditions as a percentage of unimanual task performance. Also shown are the results of one way analyses of variance (ANOVA) and covariance (ANCOVA).

For the pegboard task, the patients with schizophrenia had significantly slower performance than the controls for the unimanual and bimanual tasks. The number of pegs placed in the bimanual condition as a percentage of the unimanual condition did not differ significantly between the two groups. Similar results were found for the tapping test. While tapping bimanually the patients were significantly slower than the controls and the difference approached significance $(p=0.06)$ for unimanual tapping. But when bimanual 
tapping was considered as a percentage of unimanual performance, the two groups did not differ significantly.

Under dual task conditions of concurrent tapping and peg placement an interesting pattern of results emerged. The absolute numbers of pegs and taps were significantly different between the patients and the normal subjects, with the patients having fewer taps and placing fewer pegs in the dual task condition. The patients and controls also differed significantly in terms of percentage of change from the unimanual performance. However, relative to performance on the pegboard test alone, the patients with schizophrenia showed improved performance on the pegboard under the dual task condition, whereas the performance of the normal subjects was worse. Also, the patients with schizophrenia showed a significantly greater drop in tapping performance under dual task conditions than the normal subjects.

Under dual task conditions, for the pegboard task, we also examined the distribution of the absolute difference (pegs placed in unimanual task-pegs placed in bimanual dual task) for individual subjects in each group. This showed that peg placement in the dual task condition improved in $45 \%$, remained constant in $27 \%$, and deteriorated in $27 \%$ of the group with schizophrenia, whereas performance on the pegboard under the dual task condition improved in only $15 \%$, remained constant in $8 \%$, and deteriorated in $77 \%$ of the controls. These proportions were significantly different across the two groups $\left(\chi^{2}=5.92, d f=2, p=0.05\right)$.

When the group differences in MMS and BDI scores were covaried out, only the group differences in the unimanual pegboard remained significant.

CORRELATIONAL ANALYSIS

For the patients with schizophrenia ratings of positive symptoms $(\mathrm{r}=-0.64, \mathrm{p}<0.05)$ and negative signs $(r=-0.68, p=0.03)$ negatively correlated with bimanual pegboard placement. However, only ratings of negative signs showed significant negative correlations with pegboard placement $(\mathrm{r}=-0.72, \mathrm{p}=0.02)$ and tapping speed $(r=-0.65, p<0.05)$ in the dual task condition. The dose of neuroleptic medication did not significantly correlate with any of the measures of task performance.

\section{Discussion}

The results show that patients with schizophrenia were significantly slower than the normal controls-they placed fewer pegs and had reduced tapping speed in unimanual and bimanual conditions. However, despite this overall slowness, the decrement in bimanual performance as a percentage of unimanual performance was not significantly different for the patients and controls on either the pegboard or tapping tasks. This suggests that although motor abnormalities such as poverty of action, perseverative movements, ${ }^{14}$ and motor slowness, reflected in slower reaction time and movement time in patients than in normal controls, ${ }^{15-19}$ have been reported in schizophrenia; patients with schizophrenia do not have major deficits in bimanual coordination. By contrast, in a previous study in Parkinson's disease ${ }^{9}$ the patients showed greater decline in bimanual performance than normal subjects.

An interesting finding is that under dual task conditions, the performance of the patients with schizophrenia in peg placement actually improved relative to the unimanual pegboard task. The patients with schizophrenia were able to place more pegs while performing a secondary task with their other hand. By contrast, the tapping decreased compared with the unimanual tapping, a decrement that was significantly greater for the patients. Thus the improvement in the visually guided pegboard task was at the expense of the tapping task. Similar results have been found in patients with Parkinson's disease. ${ }^{9} 12$

Previous studies have reported that patients with schizophrenia show impaired reaction times when a simultaneous cognitive task is introduced, ${ }^{6-8}$ thus suggesting limited attentional processing capacity. Why does performance on the visually guided task improve in patients with schizophrenia under dual task conditions? Brown and Jahanshahi ${ }^{9}$ provided alternative explanations for a similar improvement obtained in patients with Parkinson's disease. The first is that for each task there may be an optimal level of attention such that too much attention is detrimental to skilled performance. For example, thinking about individual movements can impair smooth motor planning and execution, such as when running down stairs; or golfers can greatly impair their putts by thinking about each single movement in the motor sequence. It is possible that under dual task conditions, by removing some of the "excess" attention, finger tapping makes the attentional allocation to peg placement optimal for the patients so that performance on the second task improves. The second explanation is that in the light of the deficits in schizophrenia in internal generation of action with relative normality of stimulus driven behaviour ${ }^{20} 21$ rhythmical tapping acts as an external pacing cue that improves the visually guided pegboard. Thus it is possible that the patients are improving peg placement by using the rhythm of tapping as a pacing stimulus. Brown and Jahanshahi $i^{9}$ suggested that the first explanation can be tested by varying the cognitive load of a non-motor secondary task-for example, mental arithmetic-whereas the second explanation can be tested by manipulating the timing characteristics of the secondary tapping task. For patients with schizophrenia some evidence relating to these already exists. Granholm et $a l^{6}$ used a visual search task, involving pointing at a screen when a target appeared as the primary task and a simple reaction time as the secondary task. Thus the primary task, although visually guided, had a less demanding motor output component than the pegboard task in this study, and the secondary reaction time task did not involve any rhythmic cueing. The patients with schizophrenia did not improve performance on the primary visual search task 
in the dual task condition but in fact showed a greater secondary task decrement (reaction time slowing) than controls in the highest processing load dual task condition. Therefore, in the present study it is more likely that the patients are using the rhythmic finger tapping as an external pacing cue to improve performance of the visually guided task.

There is evidence that, as suggested by Frith, ${ }^{20}$ patients with schizophrenia, especially those with high ratings of negative signs, are impaired in self generated but not externally triggered movements. ${ }^{21}$ Bimanual peg placement, and tapping and peg placement under dual task conditions, were significantly associated with higher rates of negative signs, suggesting that patients with higher negative signs are more impaired under conditions in which the load on attentional capacity increases. The fact that covarying out MMS and BDI scores eliminated all significant differences between the patients and normal subjects suggests that the impairments in performance seen in the group with schizophrenia are associated with higher levels of depression and cognitive impairment.

In a recent review of evidence from various sources, Jahanshahi and Frith ${ }^{22}$ suggested that intentional or self generated actions (willed actions) are controlled differently from routine, stereotyped actions that are externally triggered by environmental stimuli. The authors also proposed that willed actions are controlled by a network of frontal cortical (dorsolateral prefrontal cortex, supplementary motor area, anterior cingulate) and subcortical (thalamus and basal ganglia) areas. Both Parkinson's disease and schizophrenia are characterised by symptoms such as akinesia or poverty of action and speech, deficits that reflect impairment of willed actions. ${ }^{22}$ Despite similarities, differences between patients with schizophrenia and Parkinson's disease are also evident. In our study of patients with Parkinson's disease an improvement for peg placement at the expense of tapping was seen, similar to that in the present study, but the patients with Parkinson's disease also showed greater impairment in the bimanual tasks than in the unimanual tasks compared with normal subjects ${ }^{9}$; which was not found for the patients with schizophrenia. The similarity in the dual task condition between the two patient groups with frontostriatal involvement, ${ }^{23}{ }^{24}$ may reflect the greater dependence of both groups on visual signals and their reliance on rhythmic tapping as an external cue to improve peg placement.

In conclusion, the results of the current study showed, relative to normal subjects, a qualitatively different pattern of motor performance in patients with schizophrenia under dual task conditions which was associated with higher ratings of negative signs.

We thank Professor Maria Ron for allowing us to study patients under her care. The financial assistance of the Wellcome Trust is gratefully acknowledged.

1 Wickens CD. Processing resources in attention. In: Parasuraman R, Davies DR, eds varieties of attention. Orlando: Academic Press, 1984:63-101.

2 Heur H, Am W. Doing two things at once: process limitations and interactions. In: Smyth MM, Wing AM, eds. The psychology of human movement. London: Academic Press, 1984:183-214.

3 Crow TJ. Molecular pathology of schizophrenia: more than one disease process? BMF 1980;280:66-8.

4 Nuechterlein KE, Dawson ME. Information processing and attentional functioning in the developmental course of

5 Norman DA, Bobrow DG. On data-limited and resourceNorman DA, Bobrow DG. On data-limited and reso
limited processes. Cognitive Psychology 1975;7:44-64.

6 Granholm E, Asarnow RF, Marder SR. Dual-task performance operating characteristics, resource limitations, and automatic processing in schizophrenia. Neuropsychology 1996;10:11-21

7 Schwartz F, Carr A, Munich RL, et al. Reaction time impairment in schizophrenia and affective illness: the role of attention. Biol Psychiatry 1989;25:540-8.

8 Schwartz F, Munich RL, Carr A, et al. Negative symptoms and reaction time in schizophrenia. $\mathcal{f}$ Psychiatr Res 1991;25:131-40.

9 Brown RGB, Jahanshahi M. An unusual enhancement of motor performance during bimanual movement in Parkinson's disease. F Neurol Neurosurg Psychiatry 1998;64:813-6. Krawiecka $M$, Goldberg D, Vaughan $M$. A standardized
psychiatric assessment scale for rating chronic psychotic psychiatric assessment scale for rating chronic
patients. Acta Psychiatr Scand 1977;55:299-308.

11 Folstein MF, Folstein SE, McHugh PR. Mini-mental state: a practical method for grading the cognitive state of patients for the clinician. F Psychiatr Res 1975;12:189-98.

12 Brown RG, Jahanshahi M, Marsden CD. The execution of bimanual movements in patients with Parkinson's, Huntington's, and cerebellar disease. F Neurol Neurosurg Psychiatry 1993;56:295-7.

13 Beck AT, Ward CH, Mendelson M, et al. An inventory for measuring depression. Arch Gen Psychiatry 1961;4:561-71.

14 Manschreck TC. Motor abnormalities in schizophrenia. In: Nasrallah HA, Weinberger DR, eds. Handbook of schizophrenia. Vol 1. Amsterdam:Elsevier, 1986:65-96.

15 Straube ER, Oades RD. Schizophrenia empirical research and Straube ER, Oades RD. Schizophrenia empiring. London: Academic Press, 1992.
finding

16 Manuzza S, Kietzman ML, Mitchell L, et al. The modality shift effect in schizophrenia: fact or artifact. Biol Psychiatry 1984;19:1317-31

17 Elkins IJ, Cromwell RL. Priming effects in schizophrenia: associative interference and facilitation as a function of visual context. F Abnorm Psychol 1994;103:791-800.

18 Nestor PG, Faux SF, McCarley RW, et al. Attentional cues in chronic schizophrenia: abnormal disengagement of attention. F Abnorm Psychol 1992;101:682-9.

19 Fuller R, Jahanshahi M. Impairment of willed actions and use of advance information for movement preparation in schizophrenia. I Neurol Neurosurg Psychiatry 1999 (in

press).
20 Frith $\mathrm{CD}$. The cognitive neuropsychology of schizophrenia. Hove: LEA, 1992.

21 Fuller R, Jahanshahi M, Nathaniel-James D. Movementrelated potentials prior to self-initiated movements are impaired in patients with schizophrenia and negative signs. Exp Brain Res 1999 (in press).

22 Jahanshahi M, Frith CD. Willed action and its impairments: Cognitive Neuropsychology 1998;15:483-533.

23 Jahanshahi M, Brown RG, Passingham R, et al. Self-initiated versus externally triggered movements: an investigation using measurement of regional cerebral blood flow and movement related potentials in normals and patients with Parkinson's disease. Brain 1995;118:913-33.

24 Weinberger DR, Berman KF, Zec RF. Physiological dysfunction of DLPC in schizophrenia. Arch Gen Psychiatary 1986;43:114-24. 